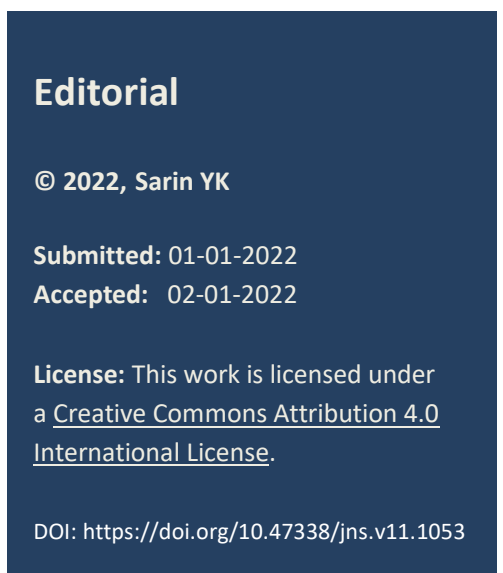

\title{
Improving neonatal surgical outcome: My vision
}

Yogesh Kumar Sarin *

President, Indian Association of Pediatric Surgeons \& Emeritus Editor-in-chief, Journal of Neonatal Surgery.

Correspondence*: Yogesh Kumar Sarin, Director Professor \& Head Department of Pediatric Surgery, Lady Hardinge Medical College, New Delhi, India. E-mail: yksarin@gmail.com

I wonder if I, President of Indian Association of Pediatric Surgeons (IAPS), or Emeritus Editor-in-chief of Journal of Neonatal Surgery (JNS), am an authority to write about the measures that would improve outcomes in surgical neonates, but my vast experience, both as a neonatal surgeon and a hospital administrator, has given me some insight in the issue and I humbly share it here. I must acknowledge that my learned friend, Prof. V Raveenthiran has provided substantial inputs. At the outset, let us admit that the outcomes in surgical neonates depend not only on the skill of the pediatric surgeon but also on that of a large team consisting of a pediatrician, anesthetist, radiologist, pathologist, biochemist, nurses, and others necessary for dealing satisfactorily with the newborn subjected to surgery.

Allow me to enumerate the Ten Commandments

1. Exclusive pediatric anesthesia team

2. Safe transport to tertiary centers

3. Inclusion of congenital anomalies under all insurance schemes

4. Instruments and medical equipment specially designed and manufactured for neonatal usage must be made available, e.g., central lines, etc.

5. Collaboration with the neonatologists

6. Peri-operative care in Surgical NICU manned by the qualified pediatric surgeon(s)

7. Audit of neonatal deaths

8. Rotating MCh postgraduates in NICU for 1-2 months during their training period

9. Strict guidelines of the infection control committee

10. A dedicated nursing team that should not be rotated to other wards

\section{Exclusive pediatric anesthesia team}

Perioperative morbidity and mortality in surgical neonates are 2-3 times higher as compared to older children and adults in our subcontinent. The anatomical, physiological, pharmacokinetic, and pharmacodynamic variations and presence of congenital cardiac, pulmonary and metabolic diseases in neonates make perioperative management challenging. [1]

Pediatric and neonatal surgery as a specialty has been in existence in India in tertiary training institutes for more than 50 years. Likewise, neonatology and neonatal intensive care facilities have also been established since long. Administering safe anesthesia to a sick surgical neonate is dependent to the greatest extent on the skill and experience of the anesthetist and to a much lesser extent on the techniques and agents selected. It is well known that children have better outcomes when anesthetized by trained pediatric anesthetists than when anesthetized by general anesthetists, [2] and it is inconceivable that we pediatric/ neonatal surgeons would be able to deliver optimal results without competent pediatric anesthetists. [3] Unfortunately, pediatric anesthesia services have failed to match up to the demand and it is only recently that training programs in pediatric and neonatal anesthesia have commenced at some centers in India; we have (National Medical Council) NMC recognized postdoctoral course (DM) in Pediatric Anesthesia running in two major autonomous government institutions and DM in Pediatric and Neonatal Anesthesia in another major public-sector medical college in Mumbai. Indian Association of Paediatric Anaesthesiologists that was founded in 2006 has now 739 members and has been responsible for starting Pediatric Anesthesia Fellowships in as many as 10 centers across India. Of course, there is still a huge gap between demand and supply, but there has been a good start in the right direction.

The practice of pediatric anesthesia requires the use of a range of equipment suitable for children of all ages. This includes specialized pediatric equipment for the administration of general as well as regional 
anesthesia, monitors, resuscitation carts, and defibrillators for the operating and recovery areas. So, first and foremost, we need to invest in pediatric anesthesia, in terms of both infrastructure and trained manpower.

\section{Safe transport to tertiary centers}

Advances in neonatal intensive care dictate that effective and efficient treatment of the sick surgical neonates can only be available by concentrating resources such as equipment and skilled staff in a few specialized tertiary neonatal surgical centers that would cater to a particular region. [4] There seems to be no justification, except in situations of dire emergency, for neonatal surgery to be performed in district general hospitals. But in the absence of quality antenatal care, many a neonate with congenital malformation(s) would be diagnosed only postnatally, and once suspected or diagnosed, they would need to be transported safely to the specialized centers, sometimes over considerable distances.

The primitive portable transportation incubators were known to exist even in the very early 20th century, but it took almost a century of improvisations before random use in some of the towns and cities of our subcontinent started. Unfortunately, a large number of surgical neonates still arrive at tertiary centres wrapped in dirty clothes traveling long distances in public transport. Most of them do not have a pretransport resuscitation or any care during transportation. [5] Hypothermia, hypoglycemia, hypoxia, and hypovolemia may all co-exist in such unfortunate neonates. All these may eventually result in fatalistic irreversible persistent pulmonary hypertension and death.

So, we need to evolve a proper referral and transport system that mandates the provision of adequate resuscitation at the referral center and the continuation of these measures during the transfer process. The surgical neonate should be accompanied by a trained junior doctor or a trained nurse. The usual transfer is done by ground ambulances, but the helicopters/planes have been also used by the corporate hospitals. One should be aware of the effect of altitude on gas-filled spaces; pneumothorax and bowel perforations may occur.

The neonate should be transferred in a transport incubator to prevent hypothermia. [6] Under ideal circumstances, facilities should be available for monitoring the infant's temperature, heart rate, and inspired oxygen concentration. Mechanical ventilation is required for a few surgical neonates, particularly for neonates with diaphragmatic hernia. Nasogastric decompression is essential for all neonates with intestinal obstruction but is also recommended for all surgical neonates to prevent vomiting and aspiration.
The tube should be aspirated at frequent intervals to ensure that the stomach is evacuated of all its contents and it should remain patent and on free drainage. Nasogastric tube and chest tube (if any) should never be clamped. Some authorities believe that nasogastric tubes are not good for decompression and ideally Replogle/ Salem sump or Blue port with or without an anti-reflux valve should be used for gastric decompression. The bowel/ viscera in patients with abdominal wall defects should be protected, and evaporative fluid loss avoided with bowel bag/ Saran wrap.

Resuscitation of the shock in the neonate should be begun at the referring hospital and should continue ideally until the neonate's condition has stabilized. There are, however, certain circumstances in which prolonged resuscitation may be counterproductive and where the delay in surgical treatment may jeopardize the infant's chances of survival (midgut volvulus, intestinal perforations, hemorrhage from a ruptured liver), and in these conditions, close collaboration with the regional tertiary center will determine the most appropriate time for the transfer. [6]

\section{Inclusion of congenital anomalies under all insurance schemes}

Indications of surgery in $80 \%$ of surgical neonates are congenital malformations. Caring for neonates with major congenital malformations has significant financial implications for the parents/caretakers as well as the treating institution, which can be positive or negative depending on whether the patient has insurance. Medical insurance is rather a new concept in this subcontinent, but it has already made inroads into the upper-middle class and the upper strata of society. Newborn baby health insurance is available in India. However, all the efforts done by IAPS in the last 2 decades to include congenital malformations in the insurance cover have not been fruitful yet. Star Health and Allied Insurance Company have been working on it and the final breakthrough is expected soon. Conditions like inguinal hernia, hypospadias shall be covered, but not complex cardiac surgery. There are few Government schemes such as Ayushman Bharat existing, but the reimbursements to the private hospitals are minuscule and so they are bound to fail. The government of India is in dialogue with IAPS for the rationalization of these reimbursements.

In high-income countries such as the USA, the reimbursement structure incentivizes non-children's hospitals to retain insured patients with congenital malformations and transfer uninsured patients with these same anomalies. This places a disproportionate financial burden on children's hospitals, while paradoxically causing insured neonates to be cared 
for at hospitals that may not be best equipped to provide complex care. [7] The true benefit of insurance will be known once it operationalizes in the subcontinent.

\section{Instruments and Medical equipment specially designed and manufactured for neonatal usage must be made available, e.g., central lines, etc.}

The reference here is not only to the smaller laparoscopic or robotic instruments but also to the instruments/ consumables such as neonatal colostomy bags, bowel bags for neonates born with gastroschisis, Replogle sumps, neonatal mosquito clamps, etc. The industry works on the principle of demand and supply, and if we continue using our local 'jugaads', these won't see the light of the day. Thanks to our neonatologist colleagues and an extensive network of NICUs, many essential items required for surgical NICU are readily available.

\section{Collaboration with neonatologists}

The care of the newborn remains unfulfilled unless there is a good sync between the neonatal surgeon and neonatologist, and both participate actively for the welfare and goodwill of the neonate. In the extremely sick patients, emergency procedures such as repair of congenital diaphragmatic hernia, resection, and anastomosis of the bowel, ligation of patent ductus arteriosus, etc. can be done bedside without the need to shift the neonate to the operation theatre provided the neonatologist can assure sound monitoring and medical management during the procedure. [8]

\section{Peri-operative care in Surgical NICU manned by the qualified pediatric surgeon(s)}

In 2012, we published a debate between neonatologists from public and private corporate hospitals about the issue of where surgical neonates should be nursed. [9] The senior neonatologist, Prof. Siddarth Ramji, who happens to be President of the National Neonatology Forum (NNF) presently, favored Surgical NICU, whereas the neonatology consultant from corporate setup favored NICU. Both had their biases and conflicts of interest. I had written an editorial for this debate and I still stand by my views a decade later. The same is reproduced verbatim here-

"Though it is recommended that all teaching pediatric surgery departments should have an independent surgical neonatal intensive care unit (NICU), only very few departments of pediatric surgery in some premiere institutes such as AIIMS New Delhi, PGIMER Chandigarh have independent surgical NICU with the desired infrastructure and personnel. The oft-quoted reasons for not having such surgical NICU include not having adequate numbers of pediatric surgical residents, lack of resources and infrastructure, inadequate exposure to the advanced gadgetries including ventilators etc. The spectrum of anomalies and diseases is so vast that it is difficult to spare time and do justice to the running of such specialized units. However, neonatal surgery is the flagship component of pediatric surgery and we should all strive to develop and maintain specialized surgical NICUs. In few countries such as Brazil and the United Kingdom, there is a provision of an additional year of training in neonatal surgery after the designated years of pediatric surgery training. The department of pediatric surgery in SMS Medical College, Jaipur has a faculty post earmarked for neonatal surgery. Such provisions will go in a long way to the advancement of neonatal surgery in this part of the world.

The other extreme of arrangement is occasionally practiced in few private corporate hospitals in metro cities of India where a neonatologist is available in the operation theatre where neonate is being operated. This appears overkill to my mind."[9]

\section{Audit of neonatal deaths}

Most of the good Surgical NICUs do continuous auditing. [10, 11] By reporting their experiences, they have encouraged other surgical NICUs to implement their regular audit meetings fitting to their improvement needs. Important areas for improvement on auditing are guideline compliance, documentation, the usage of medical devices, and clinical decisionmaking. The audit process would be successful only if there is no fear of punitive action.

\section{Rotating MCh postgraduates in NICU for 1-2 months during their training period}

We, the pediatric surgeons, can't afford to have a myopic vision that is focused on the surgical procedure at hand. Good pre-operative stabilization and postoperative management are as essential as a good surgery when it comes to surgical outcomes. Till we reach a stage where we could develop and maintain surgical NICUs, we should rotate the pediatric surgical residents to neonatology units for a period of 4-8 weeks during their training so as to sensitize them and give them enough exposure to the basics of neonatal intensive care. [9]

\section{Strict guidelines of the infection control committee}

Net death rate reported from Surgical NICU from the premier institute of the country, AIIMS, New Delhi was $14.5 \%$ and sepsis was the major reason for mortality. [10] The incidence of postoperative sepsis in GMERS Medical College, Vadodara was a whopping $73.75 \%$, i.e., every 3 out of the 4 neonates had sepsis. 
[12] These figures are even worse than the figures reported from Nigeria [13], although similar record is reported from Benin. [14]

The issue of surgical neonatal sepsis has been beautifully elaborated earlier in JNS.[15] We need to prevent sepsis by well-known strategies such as handwashing, use of disposables, continuous monitoring, and surveillance of sepsis in the Surgical NICU. Early detection and treatment with sensitive antibiotics according to each Surgical NICU's own protocol are required. Each hospital should have its monthly antibiogram reports made available so as to use appropriate antibiotics and avoid antibiotic resistance by controlling their indiscriminate use. It shouldn't be forgotten that in spite of all the research and development, no new antibiotics have been produced for years now.

The role of the hospital infection control committee is so obvious. We should strive to eradicate this preventable cause of mortality.

\section{REFERENCES}

1. Sen I, Dave N, Bhardwaj N, Juwarkar C, Beegum S. Specialised training in paediatric anaesthesia: Need of the hour. Indian J Anaesth. 2021; 65:17-22.

2. Rao KL, Batra YK. We need to develop pediatric anesthesiology in India. J Indian Assoc Pediatr Surg. 2015; 20:8-9.

3. Task force for Children's surgical care. Optimal resources for children's surgical care in the United States. J Am Coll Surg. 2014; 218:479-87.

4. Paxton JM. Transport of the surgical neonate. J Perinat Neonatal Nurs. 1990; 3:43-9.

5. Narasimhan KL, Bhaskar V. Priorities in development of neonatal surgery in India. Indian Pediatr. 2005; 42:82-3.

6. Spitz L, Wallis M, Graves HF. Transport of the surgical neonate. Arch Dis Child. 1984; 59:284-8.

7. Berman L, Rosenthal MS, Moss RL. The paradoxical effect of medical insurance on delivery of surgical care for infants with congenital anomalies. J Pediatr Surg. 2010; 45:38-43.

8. Mallick MS, Jado AM, Al-Bassam AR. Surgical procedures performed in the neonatal intensive care unit on critically ill neonates: feasibility and safety. Ann Saudi Med. 2008; 28:105-8.

9. Ramji S, Kler N, Kaur A. Where should the surgical neonates be nursed? J Neonatal Surg. 2012; 1:24.

\section{Dedicated nursing team that should not be rotated to other wards}

Trained nursing staff are an integral part of the Surgical NICU team. We need to train them and invest in their professional upliftment. These trained dedicated nurses should not be rotated to other wards. They should undergo periodic Continuous Nursing Education (CNE); the advantages of such training have been well validated previously. [16] Neonatal surgical care modules and curricula should be developed for them.

Acknowledgements: Nil

Conflict of Interest: None declared

Source of Support: Nil

Consent to Publication: No clinical figure is being used in this manuscript.

Author Contributions: Author(s) declared to fulfil authorship criteria as devised by ICMJE and approved the final version.

10. Siddharth V, Gupta SK, Agarwala S, Satpathy S, Goel P. Outcome of care provided in neonatal surgery intensive care unit of a public sector tertiary care teaching hospital of India. J Indian Assoc Pediatr Surg. 2019; 24:257-63.

11. Gangopadhyay AN, Upadhyaya VD, Sharma SP. Neonatal surgery: a ten year audit from a university hospital. Indian J Pediatr. 2008; 75:1025-30.

12. Bhatt S, Agarwal P, Patel A, Tamboli D. Audit of sepsis in neonatal surgeries at tertiary-care level hospital in India. Int J Med Sci Public Health. 2015; 4:1715-9.

13. Osifo OD, Aghahowa SEE. Audit of antibiotic therapy in surgical neonates in a tertiary hospital in Benin City, Nigeria. Afr J Paediatr Surg. 2011; 8:23-8.

14. Yassegoungbe MG, Assan BR, Houegban ASCR, Seto DM, Metchihoungbe CS, Goudjo EUEM, et al. Gastrointestinal perforations in newborns with high mortality: A series of 18 cases. J Neonatal Surg. 2020; 9:32

15. Rahman Mitul A. Surgical neonatal sepsis in developing countries. J Neonatal Surg. 2015; 4:41.

16. Laila R, Zohra J, Kashif A, Khan AA, Kauser N, Somroo R. Impact of training on nurses performance and productivity at neonatal intensive care unit (NICU). JOJ Nurse Health Care. 2018; 9:555758. 